\title{
Protective and Pathogenic Responses to Chikungunya Virus Infection
}

\author{
Kristin M. Long • Mark T. Heise
}

Published online: 24 January 2015

(C) Springer International Publishing AG 2015

\begin{abstract}
Chikungunya virus (CHIKV) is an arbovirus responsible for causing epidemic outbreaks of human disease characterized by painful and often debilitating arthralgia. Recently, CHIKV has moved into the Caribbean and the Americas resulting in massive outbreaks in naïve human populations. Given the importance of CHIKV as an emerging disease, a significant amount of effort has gone into interpreting the virus-host interactions that contribute to protection or virus-induced pathology following CHIKV infection, with the long-term goal of using this information to develop new therapies for safe and effective anti-CHIKV vaccines. This work has made it clear that numerous distinct host responses are involved in the response to CHIKV infection, where some aspects of the host innate and adaptive immune response protect from or limit virus-induced disease, while other pathways actually exacerbate the virus-induced disease process. This review will discuss mechanisms that have been identified as playing a role in the host response to CHIKV infection and illustrate the importance of carefully evaluating these responses to determine whether they play a protective or pathologic role during CHIKV infection.
\end{abstract}

Keywords Chikungunya virus $\cdot$ Alphavirus ·

Immunopathology $\cdot$ Vaccine

This article is part of the Topical Collection on Viral Tropical Medicine

K. M. Long $\cdot$ M. T. Heise

Department of Genetics, University of North Carolina at Chapel Hill, Chapel Hill, NC CB 7292, USA

K. M. Long

e-mail: kristin_long@unc.edu

M. T. Heise $(\bowtie)$

Department Microbiology and Immunology, University of North Carolina at Chapel Hill, Chapel Hill, NC CB 7292, 27599, USA

e-mail: mark_heisem@med.unc.edu

\section{Introduction}

Chikungunya virus (CHIKV) is a mosquito-transmitted alphavirus belonging to the family Togaviridae that is responsible for epidemics of debilitating rheumatic disease associated with inflammation and destruction of musculoskeletal tissues in humans [1]. CHIKV, which can be spread by the broadly distributed mosquito vectors Aedes aegypti and Aedes albopictus [2-7], has caused sporadic epidemics of infectious arthritis in Africa and Asia. Beginning in 2004, CHIKV reemerged in Africa and spread to throughout the Indian Ocean region, causing millions of infections in coastal Africa, islands within the Indian Ocean, India, and countries within Southeast Asia [2, 8-11]. In addition, infected travelers returning to northern Italy, New Caledonia, China, and the French Riviera initiated autochthonous outbreaks resulting from infection of local mosquito populations [12-16], illustrating the prominent role that infected travelers play in introducing CHIKV into new areas. This was further demonstrated by the introduction and subsequent epidemic of CHIKV into the Caribbean and the Americas in late 2013 [17-19]. As of October 17, 2014 the US Center for Disease Control and Prevention (CDC) reported a total of approximately 760,000 suspected and 14,000 confirmed cases of CHIKV in 36 countries or territories in the Caribbean, Central America, South America, and North America [20]. Furthermore, numerous cases of CHIKV have been brought back to the USA from the Caribbean, resulting in 11 instances of localized viral transmission in Florida as of October 21, 2014 [21].

The name chikungunya comes from the Makonde people of Tanzania where the virus was first identified in 1952-53 and loosely translates to "that which bends up" to describe the stooped posture of CHIKV-infected persons suffering from severe joint pain that characterizes infection. CHIKVinduced arthritis is most often symmetrical, accompanied by swelling, and involves multiple joints [22, 23]. Additionally, 
CHIKV infection is also associated with fever, headache, chills, photophobia, muscle pain, and a petechial or macropapular rash [24, 25]. Although acute CHIKV infection is generally self-limiting after $7-14$ days, continuing joint pain and lethargy are observed in about a third of patients for months, and in over $10 \%$ of patients, these sequela may persist for years [12, 23, 26-30]. Analysis of the 2004-2007 epidemic suggest that the re-emergence of CHIKV is also cause for concern due to increased morbidity and mortality associated with infection [31,32]. Greater numbers of CHIKV-infected persons developed the more severe forms of the disease including neurological complications and fulminant hepatitis, while maternal-fetal transmission associated with neonatal encephalopathy was also reported [31, 33-36].

The host immune system plays a complex role in the pathogenesis of CHIKV-induced disease. There is abundant evidence that components of the innate immune system, including the type I interferon system, play an essential role in protecting from $\mathrm{CHIKV}$-induced disease, while CHIKVspecific neutralizing antibodies mediate long-term immunity to CHIKV. However, it is also clear that components of the host immune response can also play an immunopathologic role in the pathogenesis of CHIKV-induced arthritis [37•, $38-40,41 \cdot]$. Therefore, the focus of this review is to discuss the field's current understanding of the host innate and adaptive immune response to CHIKV, with an emphasis on differentiating between those aspects of the response that mediate protection or contribute to virus-induced immune pathology.

\section{Host Cellular Processes in Response to Chikungunya Infection}

CHIKV infection at the cellular level is a cytopathic event with rapid onset of apoptosis in vivo and in vitro [42-46]. Apoptosis is thought to be the result of innate immune processes as well as the general block in host-cell translation induced by CHIKV and other alphaviruses [47-50]. Although apoptosis was suggested to be a host-protective mechanism to limit virus production and spread, it is now recognized that the host apoptotic machinery can be hijacked by many different viruses including alphaviruses to the detriment of the host [51, 52]. The release of apoptotic blebs from dying cells has been shown to increase the spread of CHIKV from apoptotic infected cells to uninfected neighboring cells as well as macrophages in vitro [53]. This process would allow for cell-tocell spread of the virus without exposure to extracellular immune cells and mediators, such as antibody. Additionally, in this system, macrophages were able to be infected following phagocytosis of CHIKV-containing blebs. Thus, apoptosis may serve as a mechanism for allowing CHIKV infection of cells that are non-permissive to direct viral entry as well as allowing the virus to evade host immune mechanisms. Finally, a recent high-throughput screen of a library of various kinase inhibitors in a human cell line identified several compounds which rather than limiting viral replication, instead decreased viral cytopathic effect, likely by targeting components of the apoptotic pathway [54]. Further work will need to be done to characterize the role for these apoptotic mechanisms and inhibitors in vivo.

Another cellular process which has been investigated in the context of alphaviruses and was shown to play both antiviral and proviral roles is the process of autophagy $[55,56]$. In particular, CHIKV infection has been shown to induce autophagy in human cells where the process dramatically increases CHIKV replication [57]. Yet in the mouse model, autophagy appears to limit CHIKV pathogenesis. Mouse embryo fibroblast (MEF) cell cultures deficient for autophagy protein (ATG) 5, a E3 ubiquitin ligase necessary for autophagy, exhibited a significant increase in CHIKV-induced cell death compared with wild-type MEFs [58]. Taken together, these studies indicate that there may be some species specificity to the autophagy mechanism and response following CHIKV infection. Indeed, experimental evidence exists indicating that the human autophagy receptor NDP52 interacts with the CHIKV non-structural protein 2 (nsP2) to promote viral replication while no such interaction occurs with the mouse orthologue of NDP52 [59•]. In addition to species specificity, these researchers showed that different autophagy receptors in human cells have distinct roles based on interactions with different viral components. In contrast to the proviral outcome of the NDP52-nsP2 interaction, the p62 autophagy receptor appears to interact with ubiquitinated CHIKV capsid protein to target it for degradation thus providing a cytoprotective mechanism for the host [59•].

Interestingly, induction of the autophagy pathway by CHIKV infection delays or mitigates the apoptotic antiCHIKV response [58]. These researchers found that CHIKV activates both the endoplasmic reticulum (ER) stress and oxidative stress pathways, albeit through distinct mechanisms, to induce autophagy. In $A \operatorname{tg} 16 \mathrm{~L}^{\mathrm{HM}}$ mice, which have decreased levels of autophagy, CHIKV infection resulted in enhanced apoptosis and subsequent increased lethality. These experiments were critical in examining the mechanisms of autophagy in CHIKV infection and elucidating a model wherein autophagy plays a protective role in CHIKV pathogenesis via its modulation of the apoptotic pathway.

Heat shock proteins (HSPs) are involved in the cellular response to stress such as protein folding/unfolding, protein transport, and protection against apoptosis. In addition, extracellular HSPs can stimulate professional antigen-presenting immune cells. HSP90 was recently shown to promote CHIK $\mathrm{V}$ infection as numerous drugs that inhibit HSP90 also inhibit viral replication and limit disease both in vitro and in a mouse model [60]. This same set of experiments identified a direct interaction between CHIKV nsP3/nsP4 and HSP90. Furthermore, HSP90 was shown to stabilize CHIKV nsP2 and promote viral replication in vitro [61]. Another protein, HSP70, 
was recently shown to bind CHIKV virus in both HEK-293 and Vero-E6 cells [62]. These studies represent the earliest examination of the role of HSPs in CHIKV infection, and it can be assumed that much more evidence of interactions between the virus and these stress response molecules will be forthcoming.

\section{Early Innate Responses to CHIKV}

The mammalian innate immune system plays a crucial role in protecting the host from viral infections, by initially sensing the virus, limiting viral replication, and ultimately shaping the nature of the adaptive immune response. Therefore, much of the work examining protective and pathologic immune responses to CHIKV have been focused on understanding how the innate immune response protects from CHIKV infection [63-65], with a significant amount of work focused on the type I IFN system and its role in protecting from CHIKVinduced disease. However, in addition to playing a protective role, there is also evidence that components of the innate immune system contribute to CHIKV-induced immune pathology. Therefore, any discussion of innate immunity's role in the pathogenesis of CHIKV-induced disease should take into account both the protective and the pathologic aspects of the disease process.

Type I Interferon-Mediated Protection From CHIKV-Induced Disease

In the vertebrate host, the type I interferon (IFN) pathway is critical for controlling viral replication and pathogenesis during the early stages of CHIKV infection. IFNs are capable of playing a direct antiviral role by limiting viral replication/ dissemination or they may regulate pro-immune cytokine expression leading to a potent host immune response. Mice deficient for both the IFN $\alpha$ and IFN $\beta$ receptor (IFNAR ${ }^{-/}$) develop severe CHIKV-induced disease associated with increased viral titers and central nervous system (CNS) involvement $[66,67]$. Given the clear importance of the IFN pathway in protecting from CHIKV-induced disease, a significant amount of work has centered on identifying points of interaction between CHIKV and the host type I IFN pathway.

In vitro work with both human peripheral blood mononuclear cells (PBMCs) and mouse dendritic cells suggests that CHIKV does not directly interact with pattern recognition receptors on hematopoietic cells to initiate the type I IFN cascade. Rather, it is cooperation between multiple host adaptor molecules on CHIKV-infected fibroblasts that appear to initiate production of type I IFN following CHIKV infection [67]. Induction of this type I IFN response occurred via the CARD adaptor inducing IFN $\beta$ (MAVS, also referred to as Cardif or IPS-1) sensor acting downstream of the RNA sensors MDA5 and RIG-I to limit CHIKV infection [67, 68].
Mouse embryo fibroblast (MEF) cultures prepared from $\mathrm{MAVS}^{-/}$animals were more sensitive to CHIKV infection than wild-type MEFS, and this correlated with a lack of IFN $\beta$ production in $\mathrm{MAVS}^{-/}$MEFS. However, MAVS ${ }^{-/-}$mice were not as sensitive to CHIKV infection as IFNAR ${ }^{-/-}$mice, suggesting that while MAVS plays a critical role in inducing a protective type I IFN response following CHIKV infection, other mechanisms are involved in inducing the type I IFN response. Indeed, the same set of experiments showed that the presence of myeloid differentiation primary response protein 88 (Myd88), the adaptor for multiple toll-like receptors (TLRs) and interleukin-1 $\beta$, may serve to limit CHIKV viral replication in vivo, presumably via type I IFN induction. The authors hypothesized that endosomal Myd88-dependent TLRs may be engaged as a result of immune cell phagocytosis of infected cells which would provide a source of viral proteins for recognition in hematopoietic cells. The fibroblast as the major type I IFN producer in CHIKV infection sets CHIK $\mathrm{V}$ apart from other closely related alphaviruses such as RRV, Sindbis virus, and VEE which are able to both infect and induce type I IFN production in mouse dendritic cells [69, 70].

Due to the critical role IFNs have in protecting against CHIKV-induced disease, many studies have focused on identifying the downstream interferon stimulated genes (ISGs) that mediate antiviral activity against CHIKV. A large-scale study which looked at gene products induced by the type I IFN in response to a wide range of viruses included CHIKV [71•]. This study identified the double-stranded RNA-specific adenosine deaminase (ADAR) as an enhancing effector for CHIK $\mathrm{V}$ replication. In contrast, multiple genes with significant inhibitory effects on CHIKV replication were also identified: SLC15A3 (Mitoferin-2), SLC25A28, HPSE (Heparanase), C6orf150 (Cyclic GMP-AMP synthase), UNC93B1 (regulates nucleotide-sensing TLRs), DDX58, (retinoic acidinducible gene 1; RIG-I), P2RY6 (Pyrimidinergic Receptor P2Y, G-Protein Coupled, 6), and interferon regulatory factor 1 (IRF-1). Of these with inhibitory effects, P2RY6, SLC15A3, and SLC25A28 were specific to CHIKV across all of the viruses tested. These findings led to screening of multiple ISGs and their contribution to CHIKV infection and disease.

Initially, expression of the ISG viperin was shown to be upregulated in human fibroblast cultures following CHIKV infection [68]. Mice deficient for viperin had higher viremia and more severe joint inflammation compared with wild-type mice following CHIKV infection [72•]. ISG15, a ubiquitinlike molecule, is protective during CHIKV infection of neonatal mice, although it was found to act in a non-classical, conjugation-independent manner [73•]. The ISG known as bone marrow stromal antigen 2 (BST-2) or tetherin was thought to act as a host restriction factor by tethering budding CHIKV virus-like particles to the host-cell membrane thus limiting viral spread [74•]. In vivo, BST-2 deficiency results 
in increased CHIKV titer at the site of inoculation leading to higher levels of viremia and increased tropism for lymphoid tissues in the face of suppressed innate inflammatory responses [75•]. However, BST-2's effects can also be antagonized by the nsP1 protein of CHIKV which is able to downregulate BST-2 expression [74•]. These findings highlight the fact that the type I IFN system is a critical protective component of the innate immune response against CHIKV infection of vertebrates, although the ability of the virus to avoid or suppress these responses prevents them from completely controlling CHIKV replication or protecting from disease $[66,67$, 73•, 76•, 77•, 78].

The Inflammatory Response to CHIKV and Mediators of Virus-Induced Immune Pathology

Although studies of the type I IFN system have provided important insights into the protective aspects of the innate immune response, there is also abundant evidence that components of the host innate and/or inflammatory response play a pathologic role during CHIKV infection. Numerous other host cells and molecules respond to protect the host and limit CHIKV replication and damage, although these responses are often poorly regulated and/or inappropriate and lead to the immune mediated pathology associated with CHIKV infection.

Macrophages appear to be the main infiltrating cell type in infected tissues following alphaviral infection [78-80, 81•, 82] and have been implicated in both protective and pathogenic mechanisms in CHIKV infection. Mice treated with clodronate liposomes to deplete macrophages showed reduced foot swelling and prolonged viremia suggesting that macrophages contribute to CHIKV-induced damage and disease, while contributing to viral control [78]. Furthermore, mice treated with bindarit, an inhibitor of monocyte chemotactic proteins (MCPs), were protected from joint and muscle tissue inflammation following CHIKV infection in a mouse model [83]. MCPs regulate macrophage migration to sites of inflammation. MCP-1 (CC chemokine 2 CCL2) is significantly elevated in early, acute CHIKV-infected human and non-human primate serum, as well as infected CHIKV mouse tissues [82, 84]. Interestingly, in mice lacking the receptor for CCL2, arthritic disease was substantially enhanced both quantitatively and temporally compared to wild-type mice without an increase in viral load or persistence [85]. In this model, the monocyte/macrophage infiltrate was replaced by a severe neutrophil and subsequent eosinophil infiltration. Thus, CCR2 ${ }^{+}$ monocytes/macrophages appear to be critical for dampening inflammation and preventing excessive musculoskeletal pathology and following CHIKV infection, potentially by limiting a more severe neutrophil-dominated response. Finally, macrophages present in CHIKV-induced musculoskeletal inflammatory lesions express genes consistent with an M2 macrophage-like activation pattern [41•]. M2 macrophages, also known as alternatively activated macrophages, are thought to have anti-inflammatory, immunoregulatory functions and exhibit a wound-healing phenotype [86, 87]. The same work demonstrated that genetic deletion of arginase 1 (Arg1), an immunoregulatory enzyme associated with M2 macrophages or macrophage-like cells, increased Ross River virus (RRV) clearance and limited tissue pathology in vivo [41•]. Therefore, M2 macrophages may actually promote the development of CHIKV persistence in some individuals, which may contribute to the development of chronic CHIK $\mathrm{V}$ disease. Furthermore, macrophages appear to be able to harbor CHIKV infection in macaques, which suggests that these cells may be responsible for the chronic symptoms observed in a percentage of human CHIKV cases [71•].

Natural killer (NK) cells are present in the inflammatory environment in many viral infections including CHIKV [78, $\left.81 \bullet, 88^{\bullet}\right]$, although their role in the disease process is unclear. In general, NK cells are one of the earliest effector cells to respond to viral infection where they exert their effects through IFN $\gamma$ production, cytokine secretion, and cytotoxic ability [83, 89]. In fact, NK cells were shown to be highly activated in the serum during human CHIKV infection, and they were not affected by the lymphopenia impacting other lymphocyte populations early after CHIKV infection [88 ${ }^{\bullet}$. $\mathrm{Ng}$ et al. demonstrated high levels of IL15, a stimulating cytokine for NK cells and T cells, in the serum of acutely infected CHIKV patients [90]. Finally, one group recently characterized the NK cell response to CHIKV infection using CHIK V-infected human cells [91]. CHIKV infection results in an early, transient shift of NK-cell phenotype and function that correlated with viral load. The study identified clonal expansion of NK cells that express both CD94/NKG2C and inhibitory receptors for HLA-C1 alleles, where these highly mature NK cells exhibited high preference for cytotoxicity coupled with diminished IFN- $\gamma$ production. However, functional impact of these cells on the CHIKV disease process is still unknown, and there is clearly a need for further explorations into the role NK cells play in CHIKV infection.

In contrast to other alphaviruses, relatively little work has been done on the role of dendritic cells (DCs) in CHIKV infection. Unlike RRV [69], Sindbis virus [92], and VEE [93], there is no evidence that CHIKV can infect DCs in humans, non-human primates or mice [42, 50, 82]. However, a study of CHIKV-infected patients who had been classified as "recovered" or "chronic" at a year postinfection identified dendritic cells as robust responders to early CHIKV infection in humans [88 $]$. Work in our lab demonstrated that the dendritic cell immunoreceptor (DCIR) plays a host-protective role in CHIKV infection of mice [64]. DCIR-deficient mouse bone marrow-derived DCs produced increased cytokines IL6 and IL10, and DCIR ${ }^{-1-}$ mice had exacerbated disease and musculoskeletal pathology following CHIKV infection. 
Additionally, there is some controversy over the ability of human blood monocytes to become infected with CHIKV. One study looked at blood samples taken from human CHIKV patients and showed that blood monocytes appear to be major targets of CHIKV infection during the viremic phase of disease [94]. The authors further supported their findings using an in vitro infection model of CHIKV infection of healthy human blood samples. In contrast, another group was not able to demonstrate infection of human peripheral blood mononuclear cells (PBMCs), as well as purified monocytes, DCs, and CD4 ${ }^{+}$T cells [42]. The discrepancies between these two experiments suggests that the involvement of these cell types in CHIKV infection may be more complicated than that observed for other alphaviruses.

\section{Adaptive Immune Response}

The role of the adaptive immune response in CHIKV clearance and pathogenesis has not been extensively studied. However, mice lacking $\mathrm{T}$ and $\mathrm{B}$ cells $\left(\mathrm{RAG}^{-/}\right.$) have persistent, high-level viremia with no evidence of inflammation within infected tissues [95•], suggesting that the adaptive immune response is critical for viral control and elimination. Additionally, Rag $1^{-/-}$mice have long-term persistent levels of CHIKV RNA in infected tissues, joints, and serum further implicating the adaptive immune response in control of CHIKV infection [39].

\section{T Lymphocytes}

In human acute CHIKV infection, acute lymphopenia has been observed [96]. $\mathrm{CD}^{+} \mathrm{T}$ cells predominate in the early stages of the disease with $\mathrm{CD} 4^{+}$T cells mediating the adaptive response at later times postinfection [65]. Both $\mathrm{CD}^{+}$and $\mathrm{CD}^{+} \mathrm{T}$ cells have been shown to infiltrate CHIKV-infected tissues in mouse models of infection [78, 81•]. While the role of $\mathrm{CD}^{+} \mathrm{T}$ cells in $\mathrm{CHIKV}$ pathogenesis remains unclear, $\mathrm{CD}^{+} \mathrm{T}$ cells were recently shown to mediate pathogenesis during CHIKV infection in mice independent of changes in viral titer and IFN $\gamma$ production [95•]. $\mathrm{CD}^{-1-}$ mice had lower levels of anti-CHIKV antibody with reduced neutralizing activity, although this did not affect their ability to control CHIK $\mathrm{V}$ infection [97]. While these studies provided evidence that $\mathrm{T}$ cells contribute to CHIKV protection and pathogenesis, characterization of the type of $\mathrm{T}$ cells responsible and the mechanism by which these cells contribute to CHIKV infection requires further study.

\section{B Lymphocytes and Antibody}

The antibody response to CHIKV has been shown to be important in human and mouse models. While T cells play a role in modulating the inflammatory response, they do not appear to play a pivotal role in limiting viral replication [95•]. On the other hand, mice that lack B cells develop viremia that persists for over a year and exhibit increased CHIKV acute disease suggestive of a role for B cells in viral clearance and control [97]. Furthermore, passive transfer of human anti-CHIKV antibody is sufficient to diminish or stop CHIKV infection in adult IFNAR ${ }^{-/}$and neonatal wild-type mice when used prophylactically [98]. In another study, combinations of mouse anti-CHIKV monoclonal antibodies were sufficient to protect IFNAR $^{-/-}$mice against lethality when given therapeutically 24-h postinfection [99]. Taken together, these findings suggest that anti-CHIKV antibodies are the major correlates of immunity and the induction of a strong $\mathrm{B}$ cell response is a critical component of CHIKV vaccine candidates.

\section{Chikungunya Vaccine Design}

Due to the explosive nature of CHIKV epidemics, vaccine design is critical from both a public health and economic standpoint. Although generally not fatal, the painful debilitating nature of CHIKV infection and its impact on productivity were sharply illustrated in the 2004-2007 outbreak. Up to $72 \%$ of patients in India suffered from arthralgia that persisted for up to 1 month following CHIKV infection [100] and within a single epidemic region, approximately $65 \%$ of disability within the population resulted from CHIKV infection [101]. However, evidence for immune-mediated pathology associated with CHIKV makes proper vaccine design imperative to avoid elements which may exacerbate disease and pathology in CHIKV-infected persons.

There have been several promising attempts to develop a vaccine against CHIKV, and while a comprehensive review of the different strategies is beyond the scope of this review, we will highlight a few examples of the different approaches that have been taken. Live attenuated CHIKV vaccines hold promise due to their relatively low production costs and their ability to elicit protective immunity with a single immunization. An attenuated CHIKV strain, designated $181 / 25$, was produced through serial passages through MRC-5 cells and tested in both mice and non-human primates and shown to offer protection against wild-type virus challenge [102]. However, in phase II trials, a small percentage of vaccinated individuals developed a mild, transient arthralgia following vaccination [103]. In addition to those adverse effects, the virus was passaged in uncertified cell cultures during production, and attenuation of the virus was found to be the result of only two point mutations [104]. In an attempt to increase the safety of attenuated vaccine viruses, another group deleted a large portion of the nsP3 gene or the entire $6 \mathrm{~K}$ gene [105]. Vaccine preparations consisted of the mutated viruses produced as viral particles or DNA-launched infectious genomes. Mice that received a single vaccination of either mutant vaccine had high levels of neutralizing antibody, a strong $\mathrm{T}$ cell response, and were 
protected against high-dose virus challenge. A second dose of the vaccine increased immunogenicity. In addition to these classical attenuation approached, another group inserted the encephalomyocarditis (EMCV) virus internal ribosome entry site (IRES) into the CHIKV subgenomic promoter, which both attenuated the virus in mammals and negated translation of the structural proteins in arthropod cells to prevent replication in mosquitoes [106]. In different mouse models, the vaccine was highly attenuated, and a single dose proved immunogenic and efficacious. Additionally, this vaccine was protective in a non-human primate challenge model [107].

One alternative to live attenuated CHIKV vaccines is the use of virus-like particles (VLPs) for vaccination. These structures mimic the organization and conformation of the authentic native virus, but lack the non-structural replication machinery and are therefore noninfectious. Noranate et al. characterized CHIKV VLPs which had been codon optimized and derived from a human cell line [108]. These VLPs were able to bind antibodies of mouse and convalescent human serum in an ELISA assay suggesting that the antigenicity of VLPs may be similar to that of wild-type CHIKV. Furthermore, CHIKV VLP vaccination of non-human primates resulted in high-titer neutralizing antibody production and protection against viremia following CHIKV challenge in both the non-human primates and mice receiving antibody transfer [109]. Additionally, the VLP vaccine was recently shown to be well tolerated by human vaccinates and elicited neutralizing antibodies which persisted for at least 6 months following a prime/boost regiment [110].

In addition to live attenuated and VLP vaccines, several groups have produced CHIKV vaccine candidates using a poxvirus vector modified vaccinia virus Ankara (MVA) expressing the CHIKV structural proteins [111]. The vaccine was highly immunogenic and protective in a mouse highdose challenge model. Other groups have used the MVA vector to express only parts of the CHIKV structural protein region and afforded protection to different mouse models against lethal challenge $[112,113]$.

\section{Conclusion}

With the introduction of chikungunya virus into the Americas, combined with the ongoing spread of the virus in Southeast Asia and the South Pacific, interest has been renewed in understanding more about this virus and the pathogenesis of CHIKV disease. Understanding the protective and pathogenic mechanisms that are initiated when virus meets host is where the game is won or lost, and information on these processes will inform the development of safe and effective CHIKV vaccines and therapeutics. It is clear from the selection of work reviewed herein that $\mathrm{CHIKV}$ disease is a complicated process involving numerous interactions between the host and virus. Furthermore, work from a number of groups indicates that some of the same processes that can protect from CHIKVinduced disease may also contribute to virus-induced pathology. Therefore, further work is clearly needed to determine how perturbations in these processes impact CHIKV-induced disease, with the long-term goal of using this information to design safer, more effective, CHIKV vaccines, or antiinflammatory therapies.

Acknowledgments Funding was provided through the Southeast Regional Centers of Excellence for Emerging Infections and Biodefense U54 AI 057157 and by U19 AI 109680.

\section{Compliance with Ethics Guidelines}

Conflict of Interest Kristin M. Long and Mark T. Heise declare that they have no conflict of interest.

Human and Animal Rights and Informed Consent For all referenced works involving animals where either Dr. Long or Dr. Heise were listed as authors, all studies were performed in accordance with the University of North Carolina at Chapel Hill Institutional Animal Care and Use Committee guidelines and with IACUC approval.

\section{References}

Papers of particular interest, published recently, have been highlighted as:

- Of importance

1. Suhrbier A, La Linn M. Clinical and pathologic aspects of arthritis due to Ross River virus and other alphaviruses. Curr Opin Rheumatol. 2004;16(4):374-9.

2. Schuffenecker I et al. Genome microevolution of chikungunya viruses causing the Indian Ocean outbreak. PLoS Med. 2006;3(7):e263.

3. Vazeille M et al. Chikungunya: a risk for Mediterranean countries? Acta Trop. 2008;105(2):200-2.

4. Vazeille $\mathrm{M}$ et al. Two Chikungunya isolates from the outbreak of La Reunion (Indian Ocean) exhibit different patterns of infection in the mosquito Aedes albopictus. PLoS One. 2007;2(11):e1168.

5. Tsetsarkin KA et al. A single mutation in chikungunya virus affects vector specificity and epidemic potential. PLoS Pathog. 2007;3(12):e201.

6. Diallo $\mathrm{M}$ et al. Vectors of Chikungunya virus in Senegal: current data and transmission cycles. Am J Trop Med Hyg. 1999;60(2): 281-6.

7. Delatte $\mathrm{H}$ et al. Geographic distribution and developmental sites of Aedes albopictus (Diptera: Culicidae) during a Chikungunya epidemic event. Vector Borne Zoonotic Dis. 2008;8(1):25-34.

8. Simon, F., H. Tolou, and P. Jeandel, [The unexpected Chikungunya outbreak]. Rev Med Interne, 2006. 27(6): p. 437 41.

9. Sergon $\mathrm{K}$ et al. Seroprevalence of Chikungunya virus (CHIKV) infection on Lamu Island, Kenya, October 2004. Am J Trop Med Hyg. 2008;78(2):333-7. 
10. Sergon $\mathrm{K}$ et al. Seroprevalence of Chikungunya virus infection on Grande Comore Island, union of the Comoros, 2005. Am J Trop Med Hyg. 2007;76(6):1189-93.

11. Laras $\mathrm{K}$ et al. Tracking the re-emergence of epidemic chikungunya virus in Indonesia. Trans R Soc Trop Med Hyg. 2005;99(2):128 41.

12. Pialoux $\mathrm{G}$ et al. Chikungunya, an epidemic arbovirosis. Lancet Infect Dis. 2007;7(5):319-27.

13. Rezza $\mathrm{G}$ et al. Infection with chikungunya virus in Italy: an outbreak in a temperate region. Lancet. 2007;370(9602):1840-6.

14. Beltrame A et al. Imported Chikungunya Infection Italy. Emerg Infect Dis. 2007;13(8):1264-6.

15. Angelini $\mathrm{P}$ et al. Chikungunya epidemic outbreak in EmiliaRomagna (Italy) during summer 2007. Parassitologia. 2008;50(1-2):97-8.

16. Angelini, R., et al., An outbreak of chikungunya fever in the province of Ravenna, Italy. Euro Surveill, 2007. 12(9): p. E070906 1.

17. Cassadou, S., et al., Emergence of chikungunya fever on the French side of Saint Martin island, October to December 2013. Euro Surveill, 2014. 19(13).

18. Leparc-Goffart I et al. Chikungunya in the Americas. Lancet. 2014;383(9916):514.

19. Van Bortel, W., et al., Chikungunya outbreak in the Caribbean region, December 2013 to March 2014, and the significance for Europe. Euro Surveill, 2014. 19(13).

20. Centers for Disease Control and Prevention. Chikungunya in the Americas. October 21, 2014 [cited 2014 October 27, 2014]

21. Centers for Disease Control and Prevention. Chikungunya virus in the United States. October 21, 2014 [cited 2014 October 27, 2014]; Available from: http://www.cdc.gov/chikungunya/geo/ united-states.html.

22. Simon $\mathrm{F}$ et al. Chikungunya infection: an emerging rheumatism among travelers returned from Indian Ocean islands. Report of 47 cases. Medicine (Baltimore). 2007;86(3):123-37.

23. Sissoko D et al. Post-epidemic Chikungunya disease on Reunion Island: course of rheumatic manifestations and associated factors over a 15-month period. PLoS Negl Trop Dis. 2009;3(3):e389.

24. Mourya DT, Mishra AC. Chikungunya fever. Lancet. 2006;368(9531):186-7.

25. Yazdani R, Kaushik VV. Chikungunya fever. Rheumatology (Oxford). 2007;46(7):1214-5. author reply 1215.

26. Fourie ED, Morrison JG. Rheumatoid arthritic syndrome after chikungunya fever. S Afr Med J. 1979;56(4):130-2.

27. Kennedy AC, Fleming J, Solomon L. Chikungunya viral arthropathy: a clinical description. J Rheumatol. 1980;7(2):231-6.

28. Brighton SW, Simson IW. A destructive arthropathy following Chikungunya virus arthritis-a possible association. Clin Rheumatol. 1984;3(2):253-8.

29. Manimunda SP et al. Clinical progression of chikungunya fever during acute and chronic arthritic stages and the changes in joint morphology as revealed by imaging. Trans R Soc Trop Med Hyg. 2010;104(6):392-9.

30. Soumahoro MK et al. Impact of Chikungunya virus infection on health status and quality of life: a retrospective cohort study. PLoS One. 2009;4(11):e7800.

31. Economopoulou A et al. Atypical Chikungunya virus infections: clinical manifestations, mortality and risk factors for severe disease during the 2005-2006 outbreak on Reunion. Epidemiol Infect. 2009;137(4):534-41.

32. Josseran $\mathrm{L}$ et al. Chikungunya disease outbreak Reunion Island. Emerg Infect Dis. 2006;12(12):1994-5.

33. Chua $\mathrm{HH}$ et al. A fatal case of chikungunya virus infection with liver involvement. Med J Malaysia. 2010;65(1):83-4.

34. Rampal M. Neurological complications in chikungunya fever. J Assoc Physicians India. 2007;55:765-9.
35. Rampal M. Hypokalemic paralysis following chikungunya fever. J Assoc Physicians India. 2007;55:598.

36. Wielanek AC et al. Guillain-Barre syndrome complicating a chikungunya virus infection. Neurology. 2007;69(22):2105-7.

37. Poo YS et al. CCR2 Deficiency Promotes Exacerbated Chronic Erosive Neutrophil-Dominated Chikungunya Virus Arthritis. J Virol. 2014. This study illustrated that complex role that specific chemokine signaling pathways play in the pathogenesis of CHIKV, which has potential implications for therapeutic development.

38. Hoarau $\mathrm{JJ}$ et al. Identical strength of the $\mathrm{T}$ cell responses against $\mathrm{E} 2$, nsP1 and capsid CHIKV proteins in recovered and chronic patients after the epidemics of 2005-2006 in La Reunion Island. PLoS One. 2013;8(12):e84695.

39. Hawman DW et al. Chronic joint disease caused by persistent Chikungunya virus infection is controlled by the adaptive immune response. J Virol. 2013;87(24):13878-88.

40. Dupuis-Maguiraga L et al. Chikungunya disease: infectionassociated markers from the acute to the chronic phase of arbovirus-induced arthralgia. PLoS Negl Trop Dis. 2012;6(3): e1446.

41. Stoermer KA et al. Genetic ablation of arginase 1 in macrophages and neutrophils enhances clearance of an arthritogenic alphavirus. J Immunol. 2012;189(8):4047-59. This study provides evidence suggesting that M2-like macrophages impact alphavirus clearance from infected tissues and the resolution of alphavirusinduced disease.

42. Sourisseau $\mathrm{M}$ et al. Characterization of reemerging chikungunya virus. PLoS Pathog. 2007;3(6):e89.

43. Dhanwani $\mathrm{R}$ et al. Characterization of chikungunya virus infection in human neuroblastoma SH-SY5Y cells: role of apoptosis in neuronal cell death. Virus Res. 2012;163(2):563-72.

44. Wikan $\mathrm{N}$ et al. Chikungunya virus infection of cell lines: analysis of the East, Central and South African lineage. PLoS One. 2012;7(1):e31102.

45. Puttamallesh VN et al. Proteomic profiling of serum samples from chikungunya-infected patients provides insights into host response. Clin Proteomics. 2013;10(1):14.

46. Dhanwani R et al. Differential proteome analysis of Chikungunya virus-infected new-born mice tissues reveal implication of stress, inflammatory and apoptotic pathways in disease pathogenesis. Proteomics. 2011;11(10):1936-51.

47. Bourai $\mathrm{M}$ et al. Mapping of Chikungunya virus interactions with host proteins identified nsP2 as a highly connected viral component. J Virol. 2012;86(6):3121-34.

48. Garmashova $\mathrm{N}$ et al. The Old World and New World alphaviruses use different virus-specific proteins for induction of transcriptional shutoff. J Virol. 2007;81(5):2472-84.

49. Toribio R, Ventoso I. Inhibition of host translation by virus infection in vivo. Proc Natl Acad Sci USA. 2010;107(21):9837-42.

50. Levine B et al. Conversion of lytic to persistent alphavirus infection by the bcl-2 cellular oncogene. Nature. 1993;361(6414):73942.

51. Griffin DE, Hardwick JM. Regulators of apoptosis on the road to persistent alphavirus infection. Annu Rev Microbiol. 1997;51: 565-92.

52. Chen YB et al. Alternate functions of viral regulators of cell death. Cell Death Differ. 2006;13(8):1318-24.

53. Krejbich-Trotot $\mathrm{P}$ et al. Chikungunya virus mobilizes the apoptotic machinery to invade host cell defenses. FASEB J. 2011;25(1): 314-25.

54. Cruz DJ et al. Identification of novel compounds inhibiting chikungunya virus-induced cell death by high throughput screening of a kinase inhibitor library. PLoS Negl Trop Dis. 2013;7(10): e2471. 
55. Eng KE et al. Accumulation of autophagosomes in Semliki Forest virus-infected cells is dependent on expression of the viral glycoproteins. J Virol. 2012;86(10):5674-85.

56. Orvedahl A et al. Autophagy protects against Sindbis virus infection of the central nervous system. Cell Host Microbe. 2010;7(2): 115-27.

57. Krejbich-Trotot $\mathrm{P}$ et al. Chikungunya triggers an autophagic process which promotes viral replication. Virol J. 2011;8:432.

58. Joubert PE et al. Chikungunya virus-induced autophagy delays caspase-dependent cell death. J Exp Med. 2012;209(5):1029-47.

59. Judith D et al. Species-specific impact of the autophagy machinery on Chikungunya virus infection. EMBO Rep. 2013;14(6):534-44. This study demonstrates that interactions between the human autophagy receptor NDP52 and the CHIKV nsP2 protein have a proviral effect by enhancing viral replication. Importantly, this effect is species specific, in that mouse NDP52 protein does not interact with CHIKV, suggesting that NSP52 may be a host range factor.

60. Rathore AP et al. Chikungunya virus nsP3 \& nsP4 interacts with HSP-90 to promote virus replication: HSP-90 inhibitors reduce CHIKV infection and inflammation in vivo. Antiviral Res. 2014;103:7-16.

61. Das I et al. Heat shock protein 90 positively regulates Chikungunya virus replication by stabilizing viral non-structural protein nsP2 during infection. PLoS One. 2014;9(6):e100531.

62. Paingankar MS, Arankalle VA. Identification of chikungunya virus interacting proteins in mammalian cells. J Biosci. 2014;39(3): 389-99.

63. Van Den Bossche, D., et al., Chikungunya virus and West Nile virus infections imported into Belgium, 2007-2012. Epidemiol Infect, 2014: p. 1-10.

64. Long KM et al. Dendritic cell immunoreceptor regulates Chikungunya virus pathogenesis in mice. J Virol. 2013;87(10): 5697-706.

65. Wauquier $\mathrm{N}$ et al. The acute phase of Chikungunya virus infection in humans is associated with strong innate immunity and T CD8 cell activation. J Infect Dis. 2011;204(1):115-23.

66. Couderc T et al. A mouse model for Chikungunya: young age and inefficient type-I interferon signaling are risk factors for severe disease. PLoS Pathog. 2008;4(2):e29.

67. Schilte $\mathrm{C}$ et al. Type I IFN controls chikungunya virus via its action on nonhematopoietic cells. J Exp Med. 2010;207(2):42942.

68. White LK et al. Chikungunya virus induces IPS-1-dependent innate immune activation and protein kinase R-independent translational shutoff. J Virol. 2011;85(1):606-20.

69. Shabman RS et al. Differential induction of type I interferon responses in myeloid dendritic cells by mosquito and mammaliancell-derived alphaviruses. J Virol. 2007;81(1):237-47.

70. Ryman KD et al. Sindbis virus translation is inhibited by a PKR/ RNase L-independent effector induced by alpha/beta interferon priming of dendritic cells. J Virol. 2005;79(3):1487-99.

71. Schoggins JW et al. A diverse range of gene products are effectors of the type I interferon antiviral response. Nature. 2011;472(7344): $481-5$.

72. Kam YW et al. Longitudinal analysis of the human antibody response to Chikungunya virus infection: implications for serodiagnosis and vaccine development. J Virol. 2012;86(23): 13005-15.

73. Werneke SW et al. ISG15 is critical in the control of Chikungunya virus infection independent of UbE1L mediated conjugation. PLoS Pathog. 2011;7(10):e1002322.

74. Jones $\mathrm{PH}$ et al. BST-2/tetherin-mediated restriction of chikungunya (CHIKV) VLP budding is counteracted by CHIK V non-structural protein 1 (nsP1). Virology. 2013;438(1):37-49.
75. Mahauad-Fernandez, W.D., P.H. Jones, and C.M. Okeoma, Critical role for BST-2 in acute Chikungunya virus infection. J Gen Virol, 2014.

76. Gardner CL et al. Interferon-alpha/beta deficiency greatly exacerbates arthritogenic disease in mice infected with wild-type chikungunya virus but not with the cell culture-adapted live-attenuated 181/25 vaccine candidate. Virology. 2012;425(2):103-12.

77. Teng TS et al. Viperin restricts chikungunya virus replication and pathology. J Clin Invest. 2012;122(12):4447-60. References 7177 demonstrate that multiple distinct interferon stimulated genes exhibit antiviral activity against CHIKV, illustrating complexity of the host innate immune effectors that contribute to CHIKV control.

78. Gardner $\mathrm{J}$ et al. Chikungunya virus arthritis in adult wild-type mice. J Virol. 2010;84(16):8021-32.

79. Heise MT, Simpson DA, Johnston RE. Sindbis-group alphavirus replication in periosteum and endosteum of long bones in adult mice. J Virol. 2000;74(19):9294-9.

80. Morrison TE et al. Characterization of Ross River virus tropism and virus-induced inflammation in a mouse model of viral arthritis and myositis. J Virol. 2006;80(2):737-49.

81. Morrison TE et al. A mouse model of chikungunya virus-induced musculoskeletal inflammatory disease: evidence of arthritis, tenosynovitis, myositis, and persistence. Am J Pathol. 2011;178(1): 32-40. This study demonstrated that CHIKV RNA persists within joints, but not other tissues, in a mouse model of CHIKV-induced arthritis.

82. Labadie $\mathrm{K}$ et al. Chikungunya disease in nonhuman primates involves long-term viral persistence in macrophages. J Clin Invest. 2010;120(3):894-906.

83. Brandstadter JD, Yang Y. Natural killer cell responses to viral infection. J Innate Immun. 2011;3(3):274-9.

84. Rulli NE et al. Protection from arthritis and myositis in a mouse model of acute chikungunya virus disease by bindarit, an inhibitor of monocyte chemotactic protein-1 synthesis. J Infect Dis. 2011;204(7):1026-30.

85. Poo YS et al. CCR2 deficiency promotes exacerbated chronic erosive neutrophil-dominated chikungunya virus arthritis. J Virol. 2014;88(12):6862-72.

86. Ruffell $\mathrm{D}$ et al. A CREB-C/EBPbeta cascade induces M2 macrophage-specific gene expression and promotes muscle injury repair. Proc Natl Acad Sci USA. 2009;106(41): $17475-80$.

87. Loke $\mathrm{P}$ et al. Alternative activation is an innate response to injury that requires $\mathrm{CD} 4+\mathrm{T}$ cells to be sustained during chronic infection. J Immunol. 2007;179(6):3926-36.

88. Hoarau JJ et al. Persistent chronic inflammation and infection by Chikungunya arthritogenic alphavirus in spite of a robust host immune response. J Immunol. 2010;184(10):5914-27. This study found that chronic CHIKV-induced arthralgia was more prevalent in individuals age 60 or older, and provides evidence CHIKV can persist over the long term within the joints of persons suffering from chronic CHIKV-induced joint disease.

89. Trifilo MJ et al. CXC chemokine ligand 10 controls viral infection in the central nervous system: evidence for a role in innate immune response through recruitment and activation of natural killer cells. J Virol. 2004;78(2):585-94.

90. Ng LF et al. IL-1beta, IL-6, and RANTES as biomarkers of Chikungunya severity. PLoS One. 2009;4(1):e4261.

91. Petitdemange $\mathrm{C}$ et al. Unconventional repertoire profile is imprinted during acute chikungunya infection for natural killer cells polarization toward cytotoxicity. PLoS Pathog. 2011;7(9): e1002268.

92. Gardner JP et al. Infection of human dendritic cells by a sindbis virus replicon vector is determined by a single amino acid substitution in the E2 glycoprotein. J Virol. 2000;74(24):11849-57. 
93. Nishimoto KP et al. Restricted and selective tropism of a Venezuelan equine encephalitis virus-derived replicon vector for human dendritic cells. Viral Immunol. 2007;20(1):88-104.

94. Her $\mathrm{Z}$ et al. Active infection of human blood monocytes by Chikungunya virus triggers an innate immune response. J Immunol. 2010;184(10):5903-13.

95. Teo $\mathrm{TH}$ et al. A pathogenic role for $\mathrm{CD} 4+\mathrm{T}$ cells during chikungunya virus infection in mice. J Immunol. 2013;190(1):259-69. This study demonstrates that CD4 T cells contribute to the development of foot sweling in a mouse model of $C H I K V$-induced arthritis.

96. Borgherini $\mathrm{G}$ et al. Outbreak of chikungunya on Reunion Island: early clinical and laboratory features in 157 adult patients. Clin Infect Dis. 2007;44(11):1401-7.

97. Lum FM et al. An essential role of antibodies in the control of Chikungunya virus infection. J Immunol. 2013;190(12):6295-302.

98. Couderc T et al. Prophylaxis and therapy for Chikungunya virus infection. J Infect Dis. 2009;200(4):516-23.

99. Pal $\mathrm{P}$ et al. Development of a highly protective combination monoclonal antibody therapy against Chikungunya virus. PLoS Pathog. 2013;9(4):e1003312.

100. Vijayakumar KP et al. Clinical profile of chikungunya patients during the epidemic of 2007 in Kerala. India J Glob Infect Dis. 2011;3(3):221-6.

101. Krishnamoorthy $\mathrm{K}$ et al. Burden of chikungunya in India: estimates of disability adjusted life years (DALY) lost in 2006 epidemic. J Vector Borne Dis. 2009;46(1):26-35.

102. Levitt $\mathrm{NH}$ et al. Development of an attenuated strain of chikungunya virus for use in vaccine production. Vaccine. 1986;4(3):157-62.

103. Edelman R et al. Phase II safety and immunogenicity study of live chikungunya virus vaccine TSI-GSD-218. Am J Trop Med Hyg. 2000;62(6):681-5.
104. Gorchakov R et al. Attenuation of Chikungunya virus vaccine strain 181/clone 25 is determined by two amino acid substitutions in the E2 envelope glycoprotein. J Virol. 2012;86(11):6084-96.

105. Hallengard D et al. Novel attenuated Chikungunya vaccine candidates elicit protective immunity in C57BL/6 mice. J Virol. 2014;88(5):2858-66.

106. Plante $\mathrm{K}$ et al. Novel chikungunya vaccine candidate with an IRES-based attenuation and host range alteration mechanism. PLoS Pathog. 2011;7(7):e1002142.

107. Roy CJ et al. Chikungunya vaccine candidate is highly attenuated and protects nonhuman primates against telemetrically monitored disease following a single dose. J Infect Dis. 2014;209(12):18919 .

108. Noranate $\mathrm{N}$ et al. Characterization of chikungunya virus-like particles. PLoS One. 2014;9(9):e108169.

109. Akahata $\mathrm{W}$ et al. A virus-like particle vaccine for epidemic Chikungunya virus protects nonhuman primates against infection. Nat Med. 2010;16(3):334-8.

110. Chang, L.J., et al., Safety and tolerability of chikungunya virus-like particle vaccine in healthy adults: a phase 1 dose-escalation trial. Lancet, 2014

111. Garcia-Arriaza J et al. A novel poxvirus-based vaccine, MVACHIKV, is highly immunogenic and protects mice against chikungunya infection. J Virol. 2014;88(6):3527-47.

112. van den Doel $P$ et al. Recombinant modified vaccinia virus Ankara expressing glycoprotein E2 of chikungunya virus protects AG129 mice against lethal challenge. PLoS Negl Trop Dis. 2014;8(9): e3101.

113. Weger-Lucarelli J et al. A novel MVA vectored chikungunya virus vaccine elicits protective immunity in mice. PLoS Negl Trop Dis. 2014;8(7):e2970. 